\title{
In Memory of Ludvig Dmitrievich Faddeev: A Giant in Mathematical Physics
}

Received: 3 May 2019 / Accepted: 21 May 2019 / Published online: 1 June 2019 (C) The Author(s) 2019

This volume of the Few-Body Systems is dedicated to the memory and life-time achievements of our colleague Ludvig Dmitrievich Faddeev who passed away on February 26, 2017. Faddeev was born on March 23, 1934 in Leningrad and followed his undergraduate university education in Leningrad University where he also received his doctorate degree of science in 1963. He spent all his professional life in the Soviet Union (later Russia). He was the head of St. Petersburg department of Steklov Institute of Mathematics of Russian Academy of Sciences from 1976 to 2000.

In the community of few-body physicists, the name "Faddeev" is synonymous with the equations which solve the quantum-mechanical problem of three interacting bodies [1]. It is remarkable that he wrote these equations down in a closed form already in 1961, when he was only 27! According to the web of science, this paper has received more than 1000 citations. However, it should remarked that many papers do not even cite this article anymore since the formalism that he developed is now a textbook item and is, very often, used as such. These equations are foundational in few-body physics. As the solution to these equations had to be carried out numerically, the first applications of his equations to solve real scattering problems did not materialize until the middle of the 80s of the last century. With the powerful computers and the development of modern two-body potentials, the field received a major boost resulting in the design of many high-precision measurements in atomic, nuclear and particle physics which could now verify the validity of the exact calculations.

He is also well known for multiple seminal contributions to theoretical physics (e.g. Faddeev-Popov ghosts [2], Faddeev-Senjanovic quantization [3,4], Faddeev-Jackiw quantization [5]).

His work has been recognized by many prizes in different countries over the past 40 years. The notable ones are: Dannie Heineman Prize (1975), Dirac Prize (1990), Max Planck Medal (1996), Pomeranchuk Prize (2002), Demidov Prize (2002), State Prize of the Russian Federation (1995, 2004), Poincaré Prize (2006), Shaw Prize (2008), and Lomonosov Gold Medal (2013).

He has been named member of several prestigious bodies, such as the Russian Academy of Sciences, the U.S. National Academy of Sciences, the French Academy of Sciences, and the Royal Society. He was the President of the International Mathematical Union from 1986 to 1990.

It was also exactly for his achievements that the international few-body physics community introduced the "Faddeev Medal" (see the picture). The medal was established in 2016 by the European Research Committee for Few-Body Problems (ERCFBP) and the American Physical Society's Topical Group on Few-Body Physics (GFB). Financial assistance from Springer was very valuable in making it a reality. During the $23^{\text {rd }}$ European Conference on Few-Body Problems in Physics held in Aarhus (Denmark) in 2016, the creation of Faddeev Medal was announced in the presence of Prof. Faddeev himself who honored the audience by his attendance at the ceremony. This new prize recognizes distinguished achievement in few-body physics. It is awarded to

This article belongs to the Topical Collection "Ludwig Faddeev Memorial Issue".

N. Kalantar-Nayestanaki $(\varangle)$

KVI-CART, University of Groningen, Zernikelaan 25, 9747 AA Groningen, The Netherlands

E-mail: n.kalantar-nayestanaki@rug.nl 
a scientist who has advanced the field significantly, either through ground-breaking research or due to crucial progress he/she achieved over the course of his/her career. The medalist is selected by an international panel of experts and the medal is awarded every three years during the International Conference on Few-body Problems in Physics. The recipients of the inaugural medal, Vitaly Efimov and Rudolf Grimm received their prize during the $22^{\text {nd }}$ International Conference on Few-Body Problems in Physics, in Caen, France in July 2018. Going through the present volume, one can encounter contributions from these two scientists.

This volume is solely dedicated to the memory of Prof. Faddeev and consists of theoretical and experimental contributions which are inspired by his work in one way or another. These range from studies of few-nucleon systems, solving Faddeev equations as well as verifying them with experimental data, to the description of the Efimov effect in atomic physics; from problems on low dimensions to hadronic physics, which are at present very exciting subjects of research. Inverse scattering problem, another facet of his work, is also addressed. In short, this memorial volume is a testament to the deep impact made by Faddeev, and even today, his foundational work still stimulates fascinating research on several fronts. Enjoy reading!
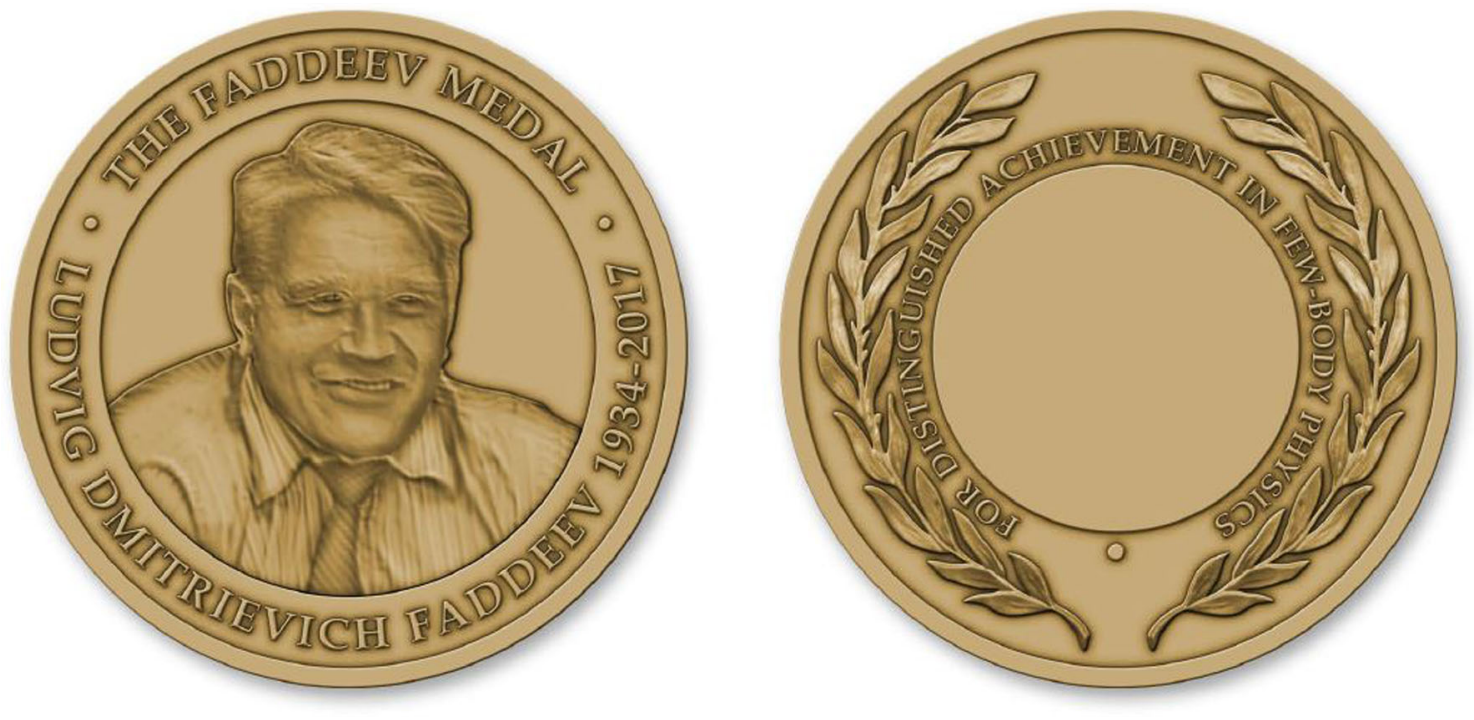

Open Access This article is distributed under the terms of the Creative Commons Attribution 4.0 International License (http:// creativecommons.org/licenses/by/4.0/), which permits unrestricted use, distribution, and reproduction in any medium, provided you give appropriate credit to the original author(s) and the source, provide a link to the Creative Commons license, and indicate if changes were made.

\section{References}

1. L.D. Faddeev, Scattering theory for a 3-particle system. Sov. Phys. JETP 12, 1014 (1961)

2. L.D. Faddeev, V. Popov, Feynman diagrams for the Yang-Mills field. Phys. Lett. B 25, 29 (1967)

3. L. Faddeev, The Feynman integral for singular Lagrangians. Theor. Math. Phys. 1, 1 (1969)

4. P. Senjanovic, Path integral quantization of field theories with second-class constraints. Ann. Phys. 100, 227 (1976)

5. L. Faddeev, R. Jackiw, Hamiltonian reduction of unconstrained and constrained systems. Phys. Rev. Lett. 60, 1692 (1988)

Publisher's Note Springer Nature remains neutral with regard to jurisdictional claims in published maps and institutional affiliations. 